\title{
Seguimento ambulatorial de prematuros: acompanhamento nutricional
}

\author{
Outpatient follow-up of preterm: nutritional monitoring
}

\author{
Ada Yukari Kanashiro Pereira ${ }^{1}$, Ciléa Maria dos Santos Ozela', Nalu de Moraes Ribeiro', \\ Ana Caroline Vieira de Almeida ${ }^{1}$, Gabriela Theresinha de Paula Souza ${ }^{1}$
}

\begin{abstract}
Resumo Objetivo: Descrever o seguimento ambulatorial de prematuros com o profissional nutricionista e apresentar o perfil nutricional destes no ambulatório de uma maternidade estadual de referência. Método: Estudo descritivo com método retrospectivo, transversal e abordagem quantitativa. Observaram-se os procedimentos realizados pelo profissional durante os atendimentos. A amostra foi de 668 pacientes, considerando os critérios de inclusão como o registro completo dos dados (número do prontuário, nome, idade, peso e estatura) e idade entre 0 a 36 meses. Excluíram-se prematuros neuropatas. Para a classificação de Peso para Idade, Estatura para Idade e Peso para Estatura, utilizaram-se as curvas da Organização Mundial da Saúde. Na análise estatística descritiva, foram utilizadas as frequências absolutas e relativas. Resultados: 50,45\% era do sexo feminino e 33,99\% encontravam-se na faixa etária de 13 a 24 meses. A maioria apresentou peso adequado para a idade (84,73\%), estatura para idade adequada $(69,16 \%)$ e peso para estatura na faixa da eutrofia $(76,50 \%)$. No entanto, $30,83 \%$ apresentaram baixa ou muito baixa estatura para a idade. Algumas das atividades desenvolvidas pelo nutricionista são relativas às orientações quanto ao aleitamento materno exclusivo, e aferição do peso e da estatura para acompanhamento do crescimento dos pacientes. Conclusão: A maioria dos prematuros encontrava-se com o peso e estatura adequados, porém uma menor e significativa parcela apresentava baixa ou muito baixa estatura para idade. Um fator limitante é a ausência de informações como idade gestacional e peso de nascimento. Este estudo poderá ser utilizado em outras pesquisas, já que há poucos acerca desta temática.
\end{abstract}

Descritores: recém-nascido prematuro; assistência ambulatorial; avaliação nutricional.

Summary Purpose: To describe the ambulatory follow-up of preterm infants with the nutritionist and to present their nutritional profile in an ambulatory of a reference state maternity. Methods: Descriptive study with retrospective, transversal method and quantitative approach. It was observed the procedures performed by the professional during the appointment. The sample was 668 patients, considering the inclusion criteria as the complete register of the data (chart number, name, age, weight and height) and age between 0 and 36 months. Preterm neuropaths were excluded. For the classification of Weight for Age, Height for Age and Weight for Height, curves of the World Health Organization were used. In the descriptive statistical analysis, the absolute and relative frequencies were used. Results: $50.45 \%$ were female and 33.99\% were in the age range of 13 to 24 months. The majority presented adequate weight for age $(84.73 \%)$, adequate height for age $(69.16 \%)$ and weight for height in the eutrophic range (76.50\%). However, $30.83 \%$ presented low or very low height for age. Some of the activities developed by the nutritionist are related to the guidelines on exclusive breastfeeding, and weight and height measurement for monitoring the growth of patients. Conclusion: The majority of preterm infants were with adequate weight and height, but a smaller and significant portion had low or very low height for age. A limiting factor is the lack of information such as gestational age and birth weight. This study may be used in other research, cause there are few on this topic.

Keywords: preterm new born; ambulatory care; nutrition assessment.

${ }^{1}$ Fundação Santa Casa de Misericórdia do Pará - FSCMPA, Belém, PA, Brasil Fonte de financiamento: Nenhuma.

Conflito de interesses: Os autores declaram não haver conflitos de interesse.

Recebido: Fevereiro 06, 2018

Aceito: Junho 01, 2018

Trabalho realizado na Fundação Santa Casa de Misericórdia do Pará - FCSMPA, Belém, PA, Brasil. 


\section{Introdução}

Atualmente, cerca de 15 milhões de nascimentos prematuros por ano são registrados no mundo todo, sendo que destes, 1,1 milhão morrem por complicações da prematuridade. Os 10 países com maior número de casos (60\% dos casos no mundo todo) são, em ordem decrescente: Índia, China, Nigéria, Paquistão, Indonésia, Estados Unidos, Bangladesh, Filipinas, República Dominicana do Congo e Brasil1 ${ }^{1}$. Considera-se recém-nascido pré-termo (RNPT) todo bebê que nasceu antes de 36 semanas e 6 dias de gestação. Quanto maior a imaturidade, maior a chance de complicações neonatais e doenças associadas que poderão surgir com o passar dos anos na vida deste prematuro².

Com o avanço no cuidado neonatal de prematuros, houve diminuição da mortalidade de RNPT com idade gestacional (IG) e peso de nascimento (PN) progressivamente menores. Cerca de $80 \%$ de RNPT têm alcançado a sobrevida devido melhorias no cuidado em terapia intensiva. Logo, a existência de ambulatórios de seguimento para atender o prematuro egresso dessas unidades de terapia intensiva é essencial para que haja o acompanhamento e avaliação de riscos que acometem o desenvolvimento a longo prazo destes prematuros $^{3}$.

O serviço ambulatorial de seguimento do prematuro é caracterizado por uma equipe multidisciplinar apta a lidar com as possíveis repercussões da prematuridade que envolvem o desenvolvimento neuromotor, metabólico e crescimento. Portanto, são avaliadas as sequelas neuromotoras e sensitivas, como a paralisia cerebral e anormalidades do desenvolvimento cognitivo, além de manifestações clínicas metabólicas como a resistência insulínica. $\mathrm{Na}$ avaliação do crescimento são avaliados os riscos para a baixa estatura, baixo peso ou déficit no crescimento do perímetro encefálico ${ }^{2}$.

Fazem parte da equipe o pediatra /neonatologista, psicóloga infantil, neurologista pediátrico, oftalmologista, otorrinolaringologista, fonoaudióloga, enfermeira, assistente social, fisioterapeuta, terapeuta ocupacional e o nutricionista clínico ${ }^{4}$.

A maternidade pesquisada possui 166 leitos para assistência ao neonato, desde a Unidade de Terapia Intensiva (UTI), unidade de cuidados intermediários (UCI) e enfermaria canguru (segunda etapa do Método Canguru). O Método Canguru (MC) é um modelo de assistência perinatal que busca, através de práticas humanizadas, o contato pele a pele do bebê e seu pais e/ou outro familiar. Surgiu em 1978 na Colômbia, e foi lançado como uma política nacional pelo Ministério da Saúde em 2000 no Brasil. Em 2015, foram registrados, em média, 800 partos. A frequência de partos prematuros foi de $38 \%$, sendo que destes, $15 \%$ foram prematuros de muito baixo peso (peso $<1.500 \mathrm{~g})^{5,6}$.

O nutricionista clínico tem como função o incentivo ao aleitamento materno, orientação sobre a introdução alimentar, manejo das crianças com falha no crescimento, e orientação nos casos de dietas específicas, como na galactosemia ${ }^{4}$. Dessa forma, esse estudo tem como objetivo descrever o atendimento nutricional do prematuro e apresentar o perfil nutricional desse público atendido no ambulatório de uma maternidade referência no estado do Pará.

\section{Métodos}

Trata-se de um estudo descritivo com método retrospectivo, transversal e abordagem quantitativa. Foi realizado em um ambulatório de seguimento do prematuro de uma maternidade de referência em atenção à gestante e recém-nascido de alto risco no estado do Pará, no período de maio a junho de 2016.

Para a descrição do atendimento nutricional foram observados os procedimentos realizados por um profissional nutricionista que fazia parte da equipe multiprofissional, como a avaliação, orientação nutricional, entre outros. Para isso, o profissional foi acompanhado durante seus atendimentos para a observação do serviço por meio de registros manuais em anotações.

Como protocolo de atendimento, os dados dos pacientes são registrados em um banco de dados próprio do Serviço de Nutrição do ambulatório. A partir desses registros, foi realizada a avaliação do perfil nutricional dos pacientes atendidos em 2015, obtendo-se uma amostra de 668 pacientes, considerando os critérios de inclusão como o registro completo dos dados (número do prontuário, nome, idade e dados antropométricos - peso e estatura) e a idade entre 0 a 36 meses ( 0 a 3 anos); e os critérios de exclusão como os prematuros com diagnóstico clínico de neuropatia, devido esse grupo apresentar outros critérios de avaliação nutricional.

O protocolo de condutas nutricionais baseava-se nas recomendações da Sociedade Brasileira de Pediatria e do Ministério da Saúde ${ }^{4,7,8}$. 
Todos os pacientes eram egressos da terceira etapa do Método Canguru, caracterizada pelo acompanhamento ambulatorial até atingir o peso de $2.500 \mathrm{~g}$.

Para a pesagem, foram utilizadas balança digital pediátrica $W e l m y^{\circledR}$, com sensibilidade de 5 g, para crianças até 24 meses e balança digital de plataforma Lider $^{\circledR}$, com sensibilidade de 100 g, para as maiores. Para aferição da estatura, utilizaram-se o antropômetro infantil para crianças até 24 meses e antropômetro vertical acoplado à balança digital para as crianças maiores segundo Plano de Frankfurt ${ }^{9}$. Ambas as medidas foram aferidas segundo as técnicas propostas pelo Ministério da Saúde 9 .

Foram utilizadas como referência para classificação de Peso para Idade (P/I), Estatura para Idade (E/I) e Peso para Estatura $(\mathrm{P} / \mathrm{E})$ as curvas da World Health Organization $(\mathrm{WHO})^{10}$. O índice antropométrico P/I indica a relação entre massa corporal e a idade cronológica da criança, muito utilizado para o acompanhamento do ganho de peso e reflete a situação atual e global da criança. $O$ índice E/I expressa o crescimento linear da criança, refletindo os efeitos acumulativos de determinadas situações sobre o crescimento da criança. $\mathrm{O}$ índice $\mathrm{P} / \mathrm{E}$ expressa a harmonia entre as dimensões da massa corporal e a estatura, podendo identificar tanto a perda de peso como o excesso de peso. Os pontos de corte utilizados para o diagnóstico nutricional da criança através do índice P/I foram os seguintes: <percentil 0,1 (muito baixo peso para idade), $\geq$ percentil 0,1 e $<$ percentil 3 (baixo peso para idade), $\geq$ percentil 3 e $\leq$ percentil 97 (peso adequado para idade) e >percentil 97 (peso elevado para idade); para o diagnóstico da E/I foram: < percentil 0,1 (muito baixa estatura para idade), $\geq$ percentil 0,1 e $<$ percentil 3 (baixa estatura para idade) e $\geq$ percentil 3 (estatura adequada para idade); e os pontos de corte para o diagnóstico do P/E foram: < percentil 0,1 (magreza acentuada), $\geq$ percentil 0,1 e $<$ percentil 3 (magreza) e $\geq$ percentil 3 e $\leq$ percentil 85 (eutrofia), $>$ percentil 85 e $\leq$ percentil 97 (risco de sobrepeso), > percentil $97 \mathrm{e} \leq$ percentil 99,9 (sobrepeso) e $>$ percentil 99,9 (obesidade). Esses índices foram considerados nessa pesquisa devido se complementarem para auxiliar na determinação do diagnóstico nutricional infantil ${ }^{9}$.

Para a análise estatística descritiva, foram utilizadas as frequências absolutas e relativas.O estudo foi aprovado pelo comitê de ética da referida maternidade sob número de protocolo 1.705.426.

\section{Resultados}

\section{Atendimento nutricional}

O atendimento nutricional é realizado por 1 nutricionista, sendo a frequência do acompanhamento na seguinte forma: mensal até o $6^{\circ}$ de vida; trimestral do $6^{\circ}$ mês ao $12^{\circ}$ mês; e semestral a partir de 12 meses.

Em cada consulta de nutrição, o profissional nutricionista realiza as aferições do peso e estatura para o acompanhamento do crescimento e desenvolvimento conforme os parâmetros estabelecidos pela WHO de $2006^{10}$. É também avaliado o ganho pôndero-estatural de acordo com a idade.

Crianças em aleitamento materno exclusivo (AME), é realizada a avaliação do manejo da amamentação quanto à posição, pega, sucção, deglutição e possíveis fatores que possam interferir na manutenção do aleitamento materno, enfatizando a importância do AME até o $6^{\circ}$ mês de idade corrigida. Para as crianças em que o AME não é possível, é realizada a orientação do manejo do uso de fórmulas infantis e do consumo adequado de água.

Para as crianças a partir do $6^{\circ}$ mês de idade corrigida é orientado o manejo da introdução alimentar, considerando o desenvolvimento da criança com base na avaliação e relatos do responsável.

Crianças que apresentam comprometimento neuromotor, como a paralisia cerebral, por exemplo, recebem orientação para adequação do aporte de calorias e nutrientes que garantam o crescimento e desenvolvimento destes.

Além disso, o ambulatório atende prematuros que apresentam casos de intolerância à lactose e alergia à proteína do leite de vaca (APLV). Destaca-se a importância do envolvimento multidisciplinar de toda a equipe, principalmente com o gastropediatra para confirmar o diagnóstico, para melhor atender estes pacientes com avaliação mais criteriosa e orientação de uma alimentação mais específica.

\section{Perfil nutricional}

Das 668 crianças, a maioria era do sexo feminino (50,45\%) e se encontravam na faixa etária de 13 a 24 meses (33,99\%). Quanto ao diagnóstico nutricional, a maioria apresentou peso adequado para a idade (84,73\%), estatura para idade adequada $(69,16 \%)$ e peso para estatura na faixa da eutrofia $(76,50 \%)$. No entanto, foi considerável o número de crianças que apresentaram baixa ou muito baixa estatura para a idade (30,83\%) (Tabela 1). 
Tabela 1. Distribuição de frequência quanto às características demográficas e antropométricas de prematuros atendidos no ambulatório de seguimento de nutrição

\begin{tabular}{lc}
\hline \multicolumn{1}{|c}{ Variáveis } & $\mathbf{N}(\%)$ \\
\hline Sexo & \\
\hline Masculino & $331(49,55 \%)$ \\
\hline Feminino & $337(50,45 \%)$ \\
\hline Idade & $157(23,5 \%)$ \\
\hline $0-6$ meses & $182(27,24 \%)$ \\
\hline $7-12$ meses & $227(33,99 \%)$ \\
\hline 13-24 meses & $102(15,27 \%)$ \\
\hline 25-36 meses & $21(3,14 \%)$ \\
\hline Peso para idade & $68(10,18 \%)$ \\
\hline Muito baixo peso & $566(84,73 \%)$ \\
\hline Baixo peso & $13(1,95 \%)$ \\
\hline Adequado & \\
\hline Elevado & $87(13,02 \%)$ \\
\hline Estatura para idade & $119(17,81 \%)$ \\
\hline Muito baixa estatura & $462(69,16 \%)$ \\
\hline Baixa estatura & $61(76,13 \%)$ \\
\hline Adequada & $33(4,94 \%)$ \\
\hline Peso para estatura & $15(2,24 \%)$ \\
\hline Magreza acentuada & \\
\hline Magreza & $(1,65 \%)$ \\
\hline Eutrofia & \\
\hline Sisco de sobrepeso & \\
\hline Obesidade & \\
\hline
\end{tabular}

\section{Discussão}

Os ambulatórios de seguimento do prematuro surgiram na década de 1970 quando neonatologistas de países desenvolvidos questionaram a evolução e o resultado a médio prazo da assistência neonatal prestada a este público. Na época, o serviço mantinha o objetivo apenas de pesquisa. No Brasil, na década de 1980, no estado do Rio de Janeiro, foram organizados ambulatórios para dar continuidade ao tratamento iniciado nas UTIs neonatais. A assistência ocorria principalmente para acompanhar o desenvolvimento neuromotor e problemas clínicos comuns dos prematuros. Atualmente, as UTIs neonatais são equipadas com tecnologias e profissionais especializados que contribuem para a sobrevida do RNPT. Isto contribuiu para o aumento da demanda em ambulatórios de seguimento, tornando-se necessário o acompanhamento de equipe multiprofissional treinada ${ }^{11}$.

Após a alta hospitalar, o aleitamento materno exclusivo deve ser recomendado. A orientação do profissional nutricionista nesta fase é relevante para que a amamentação seja mantida, pois sabe-se dos seus benefícios para o crescimento, ganho de peso, imunidade e vínculo mãe-filho. De acordo com o neurodesenvolvimento e a idade corrigida da criança, orienta-se a introdução da alimentação complementar que deve ser completa em sua composição e quantidades para suprir as necessidades do prematuro e evitar carências nutricionais. De acordo com o perfil durante a internação, alguns prematuros podem apresentar dificuldades alimentares decorrentes de situações clínicas, como enterocolite necrosante, ou uso prolongado de sonda, sendo comum os casos de reflexo de náuseas provocando vômitos, tempo prolongado para as refeições, ou ainda demanda 
frequente de alimentação de 2 em 2 horas, por exemplo. Portanto, o nutricionista deve estar apto para intervir nestas situações e garantir a nutrição do prematuro ${ }^{4}$.

O acompanhamento do crescimento e desenvolvimento é importante em todas as faixas etárias para detecção precoce de agravos à saúde. A utilização das curvas WHO de 2006 no Brasil iniciou-se a partir de 2007 por meio da sua inclusão na Caderneta de Saúde da Criança em substituição às referências do Centro Nacional de Estatística de Saúde (NCHS). Estudos relatam detecção mais precoce em casos de sobrepeso e obesidade quando se utiliza as curvas da $\mathrm{WHO}^{10,12}$.

Os resultados do presente estudo demonstraram que a maioria dos prematuros atendidos no ambulatório encontravam-se com o peso e estatura adequados. Estes resultados são semelhantes aos encontrados em alguns estudos brasileiros. Menezes et al., em seu estudo de coorte com 99 crianças nascidas com peso menor que $1750 \mathrm{~g}$, encontraram $68,4 \%$ de adequação em relação ao peso para idade de prematuros aos 6 meses de idade, atendidos em uma maternidade pública na região do Nordeste do Brasil ${ }^{13}$.

Um outro estudo de coorte, realizado também na região Nordeste com amostra de 22 crianças, avaliou prematuros com idade gestacional menor que 32 semanas. Aos 12 meses de idade, $83 \%$ encontravam-se eutróficos em relação ao peso para estatura e $11 \%$ foram diagnosticados com baixa estatura para idade ${ }^{14}$. Santos et al. ${ }^{15}$ realizaram um estudo de coorte com prematuros na cidade de Pelotas (Rio Grande do Sul). Aos 12 meses de idade $(n=179)$, foi observado que 3,4\% estavam com baixo peso, $8,7 \%$ com baixa estatura e 1,1\% com magreza. Aos 24 meses $(n=153)$, as crianças foram reavaliadas e observou-se pouca diferença com $3,0 \%$ apresentando baixo peso, $7,2 \%$ com baixa estatura e $0,8 \%$ com magreza.

O estudo realizado por Fonseca et al. na cidade de Sorocaba (São Paulo) avaliou crianças com idade gestacional menor que 34 semanas entre 16 e 24 meses de idade $(n=11)$. Também foi observado que a maioria apresentava peso para idade adequado $(81,82 \%)$ e estatura adequada para idade $(81,82 \%)^{16}$.

Rover e colaboradores referem em seu estudo o crescimento de prematuros de muito baixo peso do nascimento até 12 meses de idade corrigida, no qual as variáveis antropométricas do prematuro apresentam retardo de crescimento extrauterino na internação, porém durante o seguimento ambulatorial sofrem uma melhora progressiva nos índices ${ }^{17}$.

Apesar de a maior parte dos prematuros deste estudo ter apresentado estatura adequada para idade, é importante destacar que 30,83\% das crianças apresentaram baixa ou muito baixa estatura para a idade. Para Euser et al. ${ }^{18}$, aproximadamente $80 \%$ dos prematuros apresentam recuperação do crescimento nos primeiros 2 anos de vida. No entanto, quanto aos prematuros de muito baixo peso e de extremo baixo peso, Gaddlin afirma que estes apresentam catch-up tardio e podem continuar menores que o esperado até a adolescência ${ }^{19}$. Modi et al., ao investigarem o crescimento de crianças prematuras extremas no primeiro ano de vida, identificaram que elas terminam o primeiro ano de vida menores que crianças que nasceram à termo ${ }^{20}$.

O padrão de crescimento de RNPT no período neonatal ocorre em quatro fases. Na primeira fase, que ocorre logo após o nascimento, o recém-nascido (RN) perde peso e há retardo no crescimento devido desvio de energia para manter as funções vitais fora do ambiente uterino. Quanto mais prematuro e menor o peso de nascimento maior a perda de peso inicial. Na segunda fase, chamada de transição, com a estabilização clínica e melhor aporte calórico, há um discreto aumento do perímetro cefálico (PC) e do comprimento. Entre o $8^{\circ}$ e $24^{\circ}$ dia de vida, o RN recupera o peso de nascimento. A terceira fase, denominada catch-up ,é caracterizada por aumento acelerado de peso, comprimento e PC, ultrapassando as curvas de crescimento intra-uterino e pós-natal. Até os 12 meses, o RN recupera o PC, depois o comprimento e por último o peso. Estima-se que entre a $36^{\mathrm{a}}$ e $40^{\mathrm{a}}$ semana, atinja-se o crescimento máximo. O catch-up pode ocorrer entre os 2 e 3 anos de idade, sendo geralmente atingido no perímetro cefálico, seguido pelo comprimento e por último o peso. Na quarta fase, de equilíbrio observa-se o crescimento similar à das crianças saudáveis a termo ${ }^{21}$.

É importante considerar que o crescimento de RNPT pode variar conforme a história clínica. Portanto, é influenciado pela idade gestacional, peso de nascimento e duração de doenças iniciais. Além disso, são reconhecidos alguns fatores que aumentam o comprometimento do crescimento em RNPT: displasia broncopulmonar, enterocolitenecrosante, uso pós-natal de corticóide, hipertensão arterial durante a gestação, níveis elevados de fosfatase alcalina no período neonatal ${ }^{21}$. Um estudo realizado por Menezes et al em uma maternidade de referência no Norte do Brasil, cita o baixo peso ao nascer e a idade gestacional $\leq 36$ semanas como fatores que interferem no desenvolvimento motor, sendo importante o acompanhamento interdisciplinar precoce ${ }^{22}$.

Ainda, é importante ressaltar que a análise do ganho ponderal não deve ser dissociada do contexto familiar em que a criança está inserida, não dependendo somente das variáveis relacionadas ao período 
neonatal, como também, das condições socioeconômicas, ambiente familiar, programas de intervenção precoce para detecção, atenuação, e se possível, reversão de problemas que possam comprometer o adequado crescimento ${ }^{23,24}$. Dessa forma, é fundamental o acompanhamento do prematuro de forma supervisionada e interdisciplinar, para garantir menores taxas de novas internações, menor índice de infecções nos primeiros anos de vida e maiores e melhores taxas de crescimento e neurodesenvolvimento ${ }^{4}$.

Os resultados do presente estudo foram obtidos a partir de RNPT de 0 a 36 meses, não se limitando a idade gestacional e peso de nascimento, como mencionado nos estudos anteriores, além de não se conhecer as intercorrências clínicas no período neonatal. Logo, considera-se como fator limitante a ausência destas informações.

\section{Conclusões}

Há poucos estudos realizados com prematuros na região Norte do Brasil e há escassez de estudos que relatem o acompanhamento nutricional no follow-up, sendo este estudo uma contribuição para pesquisas na área.

Logo, o atendimento nutricional de prematuros em ambulatório especializado é importante para o acompanhamento do crescimento e desenvolvimento, garantindo a oferta de uma alimentação adequada e evitando carências nutricionais e doenças futuras.

\section{Referências}

1. World Health Organization Born too soon: the global action report on preterm birth. Geneva: WHO; 2012.

2. Brasil. Ministério da Saúde. Secretaria de Atenção à Saúde. Departamento de Ações Programáticas Estratégicas. Manual do Método Canguru: seguimento compartilhado entre a Atenção Hospitalar e a Atenção Básica. Brasília: Ministério da Saúde; 2015.

3. Castro MP, Rugolo LMSS, Margotto PR. Sobrevida e morbidade em prematuros com menos de 32 semanas de gestação na região central do Brasil. Rev Bras Ginecol Obstet. 2012;34(5):235-42. http://dx.doi.org/10.1590/S0100-72032012000500008. PMid:22584859.

4. Sociedade Brasileira de Pediatria. Manual seguimento ambulatorial do prematuro de risco. Porto Alegre: SBP; 2012.

5. Fundação Santa Casa de Misericórdia do Pará [Internet]. Relatório de gestão 2015. 2016 [citado em 10 Out. 2017]. Disponível em: http:// www.santacasa.pa.gov.br/index.php

6. Brasil. Ministério da Saude. Secretaria de Atenção à Saúde. Departamento de Ações Programáticas Estratégicas. Atenção Humanizada ao Recém Nascido de Baixo Peso: Método Canguru: manual técnico. 2. ed. Brasília: Editora do Ministério da Saúde; 2013.

7. Sociedade Brasileira de Pediatria. Manual de orientação para a alimentação do lactente, do pré-escolar, do escolar, do adolescente e na escola. 3. ed. Rio de Janeiro: SBP; 2012.

8. Brasil. Ministério da Saúde. Secretaria de Atenção à Saúde. Departamento de Atenção Básica. Dez passos para uma alimentação saudável: guia alimentar para crianças menores de dois anos: um guia para o profissional da saúde na atenção básica. 2. ed. Brasília: Ministério da Saúde; 2010.

9. Brasil. Ministério da Saúde. Secretaria de Atenção à Saúde. Departamento de Atenção Básica. Orientações para a coleta e análise de dados antropométricos em serviços de saúde: Norma Técnica do Sistema de Vigilância Alimentar e Nutricional - SISVAN. Brasília: Ministério da Saúde; 2011.

10. World Health Organization. WHO Child Growth Standards based on length/height, weight and age. Methods and development. Geneva: WHO; 2006.

11. Méio MDBB, Magluta C, Mello RR, Moreira MEL. Análise situacional do atendimento ambulatorial prestado a recém-nascidos egressos das unidades de terapia intensiva neonatais no Estado do Rio de Janeiro. Cien Saude Colet. 2005;10(2):299-307. http://dx.doi.org/10.1590/ S1413-81232005000200007.

12. Oliveira KS, Costa LCS, Silva IFC. Avaliação nutricional de crianças atendidas em ambulatório de gastroenterologia. Revista Paraense de Medicina. 2013;27(3):37-44.

13. Menezes MAS, Garcia DC, Melo EV, Cipolotti R. Recém-nascidos prematuros assistidos pelo Método Canguru: avaliação de uma coorte do nascimento aos seis meses. Rev Paul Pediatr. 2014;32(2):171-7. http://dx.doi.org/10.1590/0103-0582201432213113. PMid:25119747.

14. Santiago ACT, Oliveira LMM, Lyra PR. Perfil de crescimento de recém-nascidos prematuros menores de 32 semanas no primeiro ano de vida. Rev Ciênc Méd Biol. 2014;13(3):269-73.

15. Santos IS, Matijasevich A, Domingues MR, Barros AJD, Victora CG, Barros FC. Late preterm birth is a risk factor for growth faltering in early childhood: a cohort study. BMC Pediatr. 2009;9:71. http://dx.doi.org/10.1186/1471-2431-9-71. PMid:19917121. 
16. Fonseca MSM, Freitas PJF, Barreiros RC, Watanabe C, Gimenes CM. Avaliação do crescimento em longo prazo no recém-nascido prétermo egresso de unidade de terapia intensiva neonatal. Rev Fac Ciênc Méd Sorocaba. 2016;18(3):150-4. http://dx.doi.org/10.5327/ Z1984-4840201625613.

17. Rover MMS, Viera CS, Toso BRGO, Grassiolli S, Bugs BM. Growth of very low birth weight preterm until 12 months of corrected age. J Hum Growth Dev. 2015;25(3):351-6. http://dx.doi.org/10.7322/jhgd.90228.

18. Euser AM, de Wit CC, Finken MJ, Rijken M, Wit JM. Growth of preterm born children. Horm Res. 2008;70(6):319-28. PMid:18953169.

19. Gaddlin P. Follow-up studies of very low birthweight children in Sweden. Acta Paediatr. 2011;100(7):940-9. http://dx.doi.org/10.1111/j.16512227.2011.02288.x. PMid:21438923.

20. Modi M, Saluja S, Kler N, Batra A, Kaur A, Garg P, et al. Growth and neurodevelopmental outcome of VLBW infants at 1 year corrected age. Indian Pediatr. 2013;50(6):573-7. http://dx.doi.org/10.1007/s13312-013-0170-5. PMid:23255692.

21. Cardoso-Demartini AA, Bagatin AC, Silva RPGVC, Boguszewski MC. Crescimento de crianças nascidas prematuras. Arq Bras Endocrinol Metab. 2011;55-8.

22. Menezes LSH, Alcântara MSV, Silva ACO, Paz AC. Perfil do desenvolvimento motor em recém-nascidos pré-termos atendidos no ambulatório de follow-up. Revista Paraense de Medicina. 2013;27(1):51-5.

23. Viera CS, Mello DF, Oliveira BRG, Furtado MC. Rede e apoio social familiar no seguimento do recém-nascido pré-termo e baixo peso ao nascer. Revista Eletrônica de Enfermagem. 2010;12:11-9.

24. Viera CS, Rech R, Oliveira BRB, Maraschin MS. Seguimento do pré- termo no primeiro ano de vida após alta hospitalar: avaliando o crescimento pondoestatural. Revista Eletrônica de Enfermagem. 2013;15(2):407-15.

\footnotetext{
Autor correspondente

Ada Yukari Kanashiro Pereira

Av. Magalhães Barata, 1032

CEP 66060-281, Belém, PA, Brasil

Tel.: (91) 98289-6508

E-mail: adaykanashiro@gmail.com

Informação sobre os autores

AYKP, NMR, ACVA e GTPS são nutricionistas; especialistas em Atenção a Saúde da Mulher e da Criança pelo Programa de Pós-graduação em Residência Multiprofissional da Universidade do Estado do Pará (UEPA).

CMSO é nutricionista da Fundação Santa Casa de Misericórdia do Pará (FSCMPA); mestre em Gestão de Empresas pela Universidade Lusófona de

Humanidades e Tecnologias.

Contribuição dos autores
}

Todos os autores contribuíram para a concepção do trabalho, da análise e interpretação dos dados, de sua redação e revisão crítica.

Todos os autores leram e aprovaram a versão final submetida ao Pará Research Medical Journal. 